\title{
Numerical Scheme for Dynamic Response of Deep-water Risers
}

\author{
Carlos RIVEROS ${ }^{1}$, Tomoaki UTSUNOMIYA ${ }^{2}$, Katsuya MAEDA ${ }^{3}$ and Kazuaki ITOH $^{4}$ \\ ${ }^{1}$ Student Member, M. Eng., Dept. of Civil and Earth Resources Eng., Kyoto University \\ (Yoshida-honmachi, Sakyo-ku, Kyoto 606-8501, Japan) \\ ${ }^{2}$ Member, Dr. Eng., Associate Professor, Dept. of Civil and Earth Resources Eng., Kyoto University \\ (Yoshida-honmachi, Sakyo-ku, Kyoto 606-8501, Japan) \\ ${ }^{3}$ Ph.D., Deep Sea Technology Research Group, National Maritime Research Institute \\ (6-38-1, Shinkawa, Mitaka-shi, Tokyo 181-0004, Japan) \\ ${ }^{4}$ Dr. Eng., Deep Sea Technology Research Group, National Maritime Research Institute \\ (6-38-1, Shinkawa, Mitaka-shi, Tokyo 181-0004, Japan)
}

\begin{abstract}
A numerical scheme for dynamic response of deep-water risers is presented in this paper; its formulation is based on the Finite Element Method (FEM) and the quasi-steady model for prediction of the transverse forces. The increased mean drag coefficient during lock-in is also considered in the numerical scheme. The simulation results are compared to experimental data obtained from a 35-meter long flexible riser model. Good agreement is observed in these comparisons. The in-line response of the riser model is well represented by the numerical scheme and the transverse response is under-predicted as the oscillating frequency increases.
\end{abstract}

Key Words: deep-water riser, vortex-induced vibrations, quasi-steady model, Morison equation.

\section{Introduction}

There is an increasing interest in developing floating offshore facilities for deep petroleum production. One of the main challenges of such developments is the riser system employed to transport oil from the seabed to floating offshore structures. The main concern for designers is that strong marine currents may induce vibrations created by vortices shed from the riser. These Vortex-Induced Vibrations (VIV) may cause severe fatigue damage to the riser. The resonant type VIV, when the vortex shedding frequency approaches, or is coincident with, a natural frequency of the riser, may cause considerable transverse oscillations of the riser.

The VIV analysis of a deep-water riser is still challenging due to the fact that the riser can be excited along its length in different modes and at different frequencies leading to a modal response dominated by mode interference, multi-mode response, mode switching and frequency dependence of the added mass. Several analytical models have been developed to describe the dynamic response of deep-water risers; these models predict quite well the dynamic response of risers under controlled experimental conditions having many difficulties when they are dealing with severe environmental conditions as reported by Hong and Koterayama ${ }^{1)}$ and Faccinetti et al. ${ }^{2)}$.

Numerical methods have been extensively used to solve the coupled problem of VIV of risers. There are basically three methods, namely the direct numerical simulation, the Vortex-In-Cell (VIC) method and the Finite Element Method (FEM) ${ }^{3}$. The numerical approach has many limitations considering the large number of variables that must be included in the analysis; in addition, modal response in sheared current is still not well understood. The fluid motion and the motion of the riser must be coupled in order to obtain a good prediction model; especially in the lock-in region, where the vortex shedding frequency collapses onto the natural frequency. It is still challenging to numerically predict the dynamic behavior of this coupled system. Most of the numerical simulations are restricted to the lower end of the Reynolds number spectrum. 
The semi-empirical approach is also widely used to predict the VIV response of risers. The current semi-empirical prediction programs used large databases of experimentally determined coefficients to predict VIV. Although these programs are widely used for practical applications, different models for the prediction of VIV can give different results among these programs as reported by Gabbai and Benaroya $^{3}$. Several experiments have also been conducted recently to better understand the VIV response of risers. Some of these experiments were carried out to measure the hydrodynamic input coefficients for the aforementioned semi-empirical prediction programs to validate analytically derived models of risers. The main conclusion that can be drawn from these experiments is that VIV response is an inherently nonlinear, self-regulated, multi-dof phenomenon ${ }^{3)}$.

A numerical scheme to simulate the VIV response of long flexible risers is presented in this paper. The Finite Element Method (FEM) is used in conjunction with the quasi-steady model to predict the transverse response of the riser taking into account the main features of the VIV process. Experimental validation is also carried out using a 35-meter flexible riser model.

The main objective of the development of this numerical scheme is to provide a reliable tool that can be used to implement vibration-based damage detection methodologies in deep-water risers. It is still challenging such implementations, but the first step in this direction must be the development of time-efficient and accurate dynamic response tools.

\section{Numerical Scheme}

One of the most widely used models to describe the in-line motion of a structure excited by a fluid flow is presented in Eq. (1) ${ }^{4}$.

$$
\begin{aligned}
& m_{0} \ddot{x}_{1}+2 m_{0} \zeta_{0} \omega_{0} \dot{x}_{1}+k_{x 1} x_{1}=\rho S C_{m} \dot{U}_{1} \\
& -\rho S C_{i} \ddot{x}_{1}+\frac{1}{2} \rho D C_{d}\left(U_{1}-\dot{x}_{1}\right)\left|U_{1}-\dot{x}_{1}\right|
\end{aligned}
$$

where $m_{0}$ is the mass of the structure per unit length, $x_{1}$ is the relative displacement of the structure, $\zeta_{0}$ is the damping coefficient, $\omega_{0}$ is the natural frequency of the structure without fluid surrounding it and $k_{x l}$ is the stiffness parameter related to the combination of the physical constants of the structure such as Young's modulus and Poisson's ratio and its moments of the inertia. The density of the surrounding fluid is denoted by $\rho$, the crosssectional area of the displaced fluid by $S$, the steady velocity of the fluid in the in-line direction acting on the surface of the structure is defined by $U_{l}$, and $D$ is defined as the characteristic length (e.g. diameter of the riser). The three force parameters correspond to the widely recognized approach proposed by Morison et al.5). The mean drag coefficient is denoted by $C_{d}$, the added-mass coefficient by $C_{i}$ and the inertia coefficient is defined by $C_{m}=C_{i}+1.0$.

\subsection{The Quasi-Steady Model}

The analytical representation of the transverse lift force is incorporated into the numerical scheme by using the quasi-steady procedure presented by Obasaju et al.6). This model can be used in conjunction with the left-hand side of the Eq. (1) to represent the cross-flow response of the structure using the corresponding stiffness parameter and the relative displacement of the structure in the cross-flow direction. The quasi-steady procedure assumes that regular shedding of vortices produces a sinusoidal force (transverse lift force), which is proportional to the square of the in-line maximum velocity as shown in Eq. (2).

$$
F_{L}(t)=\frac{1}{2} \rho U_{0}^{2} D C_{L \max } \sin \left(2 \pi t \omega_{L}+\psi\right)
$$

where $F_{L}$ is the lift force per unit length of the structure, $U_{0}$ is the relative in-line maximum velocity, $C_{L \max }$ is the maximum lift coefficient $\left.{ }^{6}\right), \omega_{L}$ is the dominant frequency ${ }^{6}$ ) and $\psi$ is the phase angle. The dominant frequency mainly depends on the Keulegan-Carpenter number (KC) when the Strouhal number $S_{t}=0.2$ (Reynolds Numbers $2.5 \times 10^{2}$ $<\operatorname{Re}<2.5 \times 10^{5}$ ). Obasaju et al. $\left.{ }^{6}\right)$ showed that the vortex patterns around a circular cylinder in oscillating flow can be approximately divided into five regimes, namely the asymmetric $(4 \leq \mathrm{KC} \leq$ $8)$, the transverse $(8 \leq \mathrm{KC} \leq 15)$, the diagonal $(15 \leq \mathrm{KC} \leq 22)$, the third vortex $(22 \leq \mathrm{KC} \leq$ $30)$, and the quasi-steady ( $\mathrm{KC} \geq 30$ ). Each of these regimes is characterized by an approximate dominant frequency.

The basic assumption behind the quasi-steady procedure is that static fluid forces measured on a stationary body can be used to approximate dynamic fluid forces on an oscillating body, the main drawback of this approach is that fluid-structure interaction is completely neglected ${ }^{7}$.

One of the major challenges in the numerical implementation of the quasi-steady model in deep-water risers is the correct calculation of the phase angle, $\psi$. Each section of a deep-water riser is excited at a particular dominant frequency in the 
cross-flow direction, which indeed is related to its in-line amplitude; therefore, the phase angle used in Eq. (2) must be calculated only when the steady response is achieved; otherwise, wrong in-line amplitudes obtained during the transient response may under-estimate the phase angle and lead to out-of-phase response between the in-line and the cross-flow motions of the deep-water riser.

\subsection{Increased Mean Drag Coefficient Model}

Vandiver ${ }^{8)}$, using experimental data, showed that mean drag coefficients in excess of three can be achieved when the vortex shedding frequency approaches one of the natural frequencies of a oscillating body, this phenomena is called lock-in. Experimental studies have shown that lock-in occurs when the reduced velocity, $\left(U_{r}=U_{1} /\left(f_{\text {osc }} D\right)\right)$, reaches a value between 4 and $8^{7}$ ). Here, $f_{o s c}$, is the oscillating frequency of the body.

Kim and Perkins ${ }^{9)}$ presented a methodology to compute the increased mean drag coefficient during lock-in using the maximum peak-to-peak amplitude $(2 \bar{Y})$ in the cross-flow direction (transverse direction). The increased mean drag coefficient is then computed from Eq. (3), where $C_{d o}$ is the mean drag coefficient and $C_{d}$ is the increased mean drag coefficient.

$$
\begin{aligned}
& \frac{C_{d}}{C_{d 0}}=\left\{\begin{array}{l}
1, w_{r}<1 \\
1+1.16\left(w_{r}-1\right)^{0.65}
\end{array}\right. \\
& w_{r}=\left(1+2 \frac{\bar{Y}}{D}\right)\left(U_{r} S_{t}\right)^{-1}
\end{aligned}
$$

\section{Numerical Implementation}

The numerical solution of the differential equation governing the static and dynamic behavior of a flexible riser, presented in Eq. (1), is carried out using the Finite Element Method (FEM). The commercial software ABAQUS ${ }^{10}$ is used to assemble the FE model of the riser and the ABAQUS/Aqua ${ }^{10)}$ capability is used to input the in-line hydrodynamic forces to the riser.

The riser is then idealized as an assembly of 2-node cubic pipe elements using the EulerBernoulli beam theory. The main idea behind this procedure is that using multiple beam elements to compose the flexible riser allows the element cubic shape functions to more closely fit the actual shape function of a nonlinear beam, thus improves the simulation accuracy.
Due to the inherently nonlinear behavior of the riser response, a nonlinear time-domain method is selected. A static stress analysis is performed in order to apply the self-weight of the riser. As a result, the geometric nonlinearity is included during this step. The dynamic response of the riser is computed using the direct-integration method ${ }^{10)}$, during this step the quasi-steady model and the increased mean drag coefficient model are incorporated into the proposed numerical scheme using an in-house FORTRAN subroutine developed by the authors.

\subsection{Free-end Riser Models}

The numerical scheme is first implemented in free-end riser models, these models are sinusoidal excited at their top end and free supported at their bottom end. Hong and Koterayama ${ }^{1}$ developed a new analytical scheme for dynamic response of flexible risers under controlled environmental conditions and compared the results obtained from their analytical scheme with experimental data. Transverse force was not considered in the numerical scheme ${ }^{1)}$. The experimental model ${ }^{1)}$ has a length of $6.5 \mathrm{~m}$, Young's modulus of $8.847 \mathrm{MPa}$, outer diameter of $0.0225 \mathrm{~m}$, inner diameter of $0.0127 \mathrm{~m}$ and density of $1476 \mathrm{~kg} / \mathrm{m}^{3}$. A bottom weight is added to the model in order to keep it straight during the dynamic tests. This bottom weight has a diameter of $0.034 \mathrm{~m}$, a total length of $0.093 \mathrm{~m}$ and a weight in water of $3.489 \mathrm{~N}$.

The experimental riser model presented by Hong and Koterayama ${ }^{1)}$ is simply supported at its top end and free supported at its bottom end. The riser model is excited at its top-end by a sinusoidal forced oscillation motion with amplitude of $0.1 \mathrm{~m}$ and forced oscillation periods of $2 \mathrm{sec}$., $6 \mathrm{sec}$. and $10 \mathrm{sec}$. At the water level there is no horizontal velocity component $(U=0)$. The riser model is excited at Reynolds numbers (Re) up to 2000 and Keulegan-Carpenter (KC) numbers up to 28 . This regime is named the third vortex $(22 \leq \mathrm{KC} \leq$ 30 ) by Obasaju et al. ${ }^{6}$. In this regime three full vortices are formed during each half cycle and three vortex pairs convect away during a complete cycle.

The forced oscillation experiments are carried out in an experimental tank of $65 \mathrm{~m}$ long, $5 \mathrm{~m}$ wide and $7 \mathrm{~m}$ deep. Ten CCD cameras are used to measure the motion of the riser model; each pair of cameras is arranged at the same level in the $x-y$ direction. Fig.1 depicts the riser motion, where the $x$-axis is defined in the direction of the forced oscillation motion. The coordinate system is then defined using the $z$-axis in the direction of the riser's axis. 


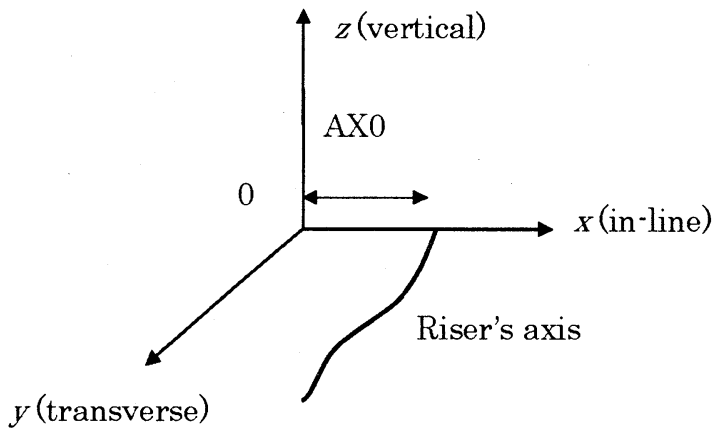

Fig.1 Riser Motion and Coorditate System.

The analytical and experimental results presented by Hong and Koterayama ${ }^{1)}$ are shown in Fig.2. The horizontal axis shows the in-line displacements, which are normalized by the amplitude of the forced oscillation motion (AX0). Fig.3 shows the simulation results obtained with the proposed numerical scheme. Good agreement is observed.

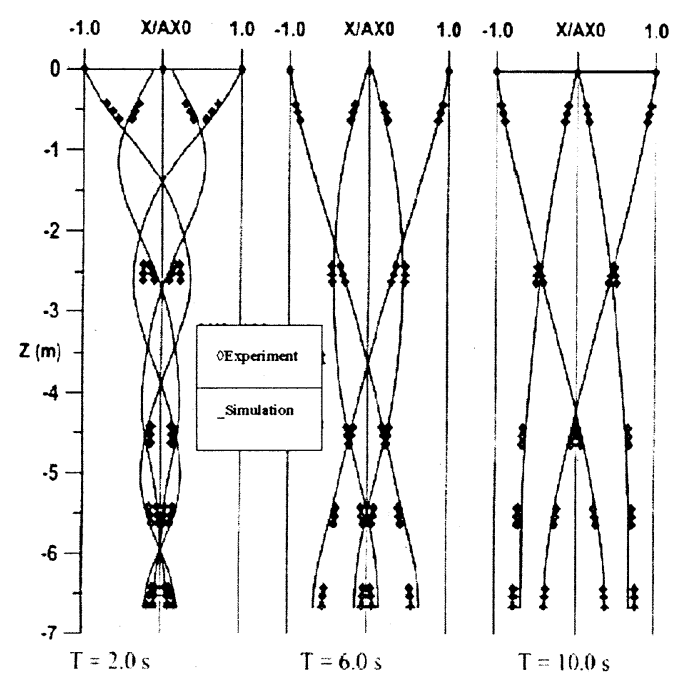

Fig. 2 In-line Response (Hong and Koterayama ${ }^{1}$ ).

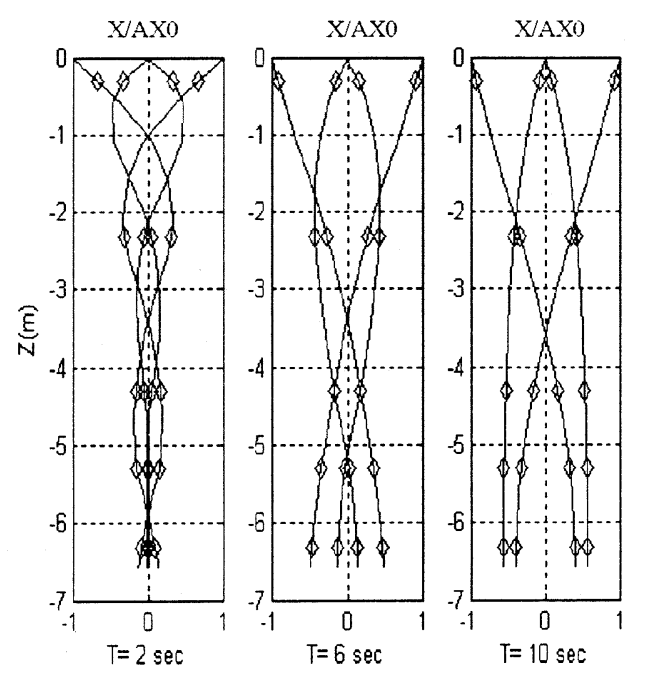

Fig.3 In-line Response (Proposed Numerical Scheme).
A second riser model, using the same properties and boundary conditions from the model previously presented ${ }^{1)}$, was developed by Senga and Koterayama ${ }^{11}$. The previous numerical scheme ${ }^{1)}$ is extended by Senga and Koterayama ${ }^{11)}$ in order to include the transverse (lift) force using the harmonic model presented in Eq. (4).

$$
F_{L}(t)=\frac{1}{2} \rho U^{2} D C_{L} \sin \left(2 \pi t f_{s}+\psi\right)
$$

where $F_{L}$ is the lift force per unit length of the structure, $U$ is the instant in-line velocity, $C_{L}$ is the lift coefficient, $f_{s}$ is the shedding frequency and $\psi$ is the phase angle. The experimental riser model $^{11)}$ is excited by a sinusoidal force oscillation motion with amplitude of $0.1 \mathrm{~m}$ and force oscillation period of $8 \mathrm{sec} .{ }^{11)}$.

According to Senga and Koterayama ${ }^{11)}$ the oscillation parameters avoid the lock-in condition, because the oscillating frequency is located in between the nearest natural frequencies of the model. The riser model developed by Senga and Koterayama ${ }^{11)}$ has fundamental periods of $11 \mathrm{sec}$., $4.8 \mathrm{sec}$., $3.1 \mathrm{sec}$. and $2.3 \mathrm{sec}$.

The analytical and experimental results obtained from the riser model presented by Senga and Koterayama ${ }^{11}$, using the transverse response of the riser model at a depth of $4.31 \mathrm{~m}$ (YC2), are shown in Fig.4. The simulation results obtained by the proposed numerical scheme are shown in Fig.5. In the proposed numerical scheme the hydrodynamic coefficients were obtained from experiments conducted by Koterayama and Nakamura ${ }^{12)}$, and the maximum lift coefficients were obtained from Sanghafian et al. ${ }^{13)}$.

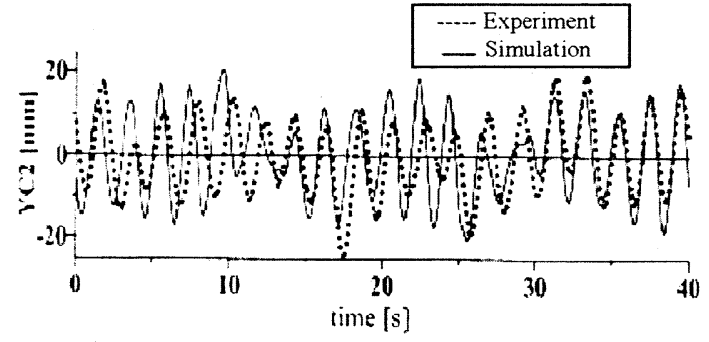

Fig.4 Transverse Response (Senga and Koterayama ${ }^{11}$ ).

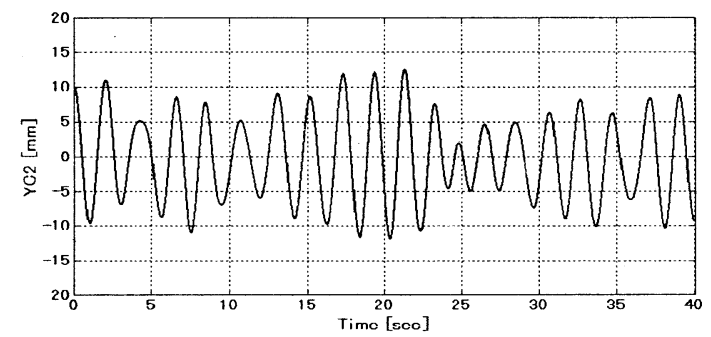

Fig.5 Transverse Response (Proposed Numerical Scheme). 
The in-line response of the riser model is accurately represented by the proposed numerical scheme as shown in Fig.2 and 3. On the other hand, it can be seen from Fig.5 that the transverse response at YC2 during 40 seconds has the same frequency content of the experimental data presented in Fig.4 resulting in 19 positive peaks; therefore, the quasi-steady model using the dominant frequency appropriately represents the transverse motion at $\mathrm{YC} 2$. Some differences are observed in the maximum displacements of the transverse response at $\mathrm{YC} 2$ due to the inherently nonlinear nature of the VIV process. Senga and Koterayama $^{11)}$ reported that the error between the experimental and simulations results is mainly induced by the direction of the VIV.

\subsection{Long Flexible Riser Model}

\subsubsection{Experimental Model}

The forced oscillation experiments are carried out in the deep-sea basin of the Integrated Laboratory for Marine Environmental Protection located in the National Maritime Research Institute (NMRI). This deep-sea basin is shown in Fig.6 and consists of two main parts, a circular basin (depth: 5 $\mathrm{m}$, effective diameter: $14 \mathrm{~m}$ ) and a deep pit (depth: $30 \mathrm{~m}$, effective diameter: $6 \mathrm{~m}$ ). The underwater 3-dimensional measurement equipment consists of 20 high-resolution digital cameras ( 2 units/set x 10 sets).

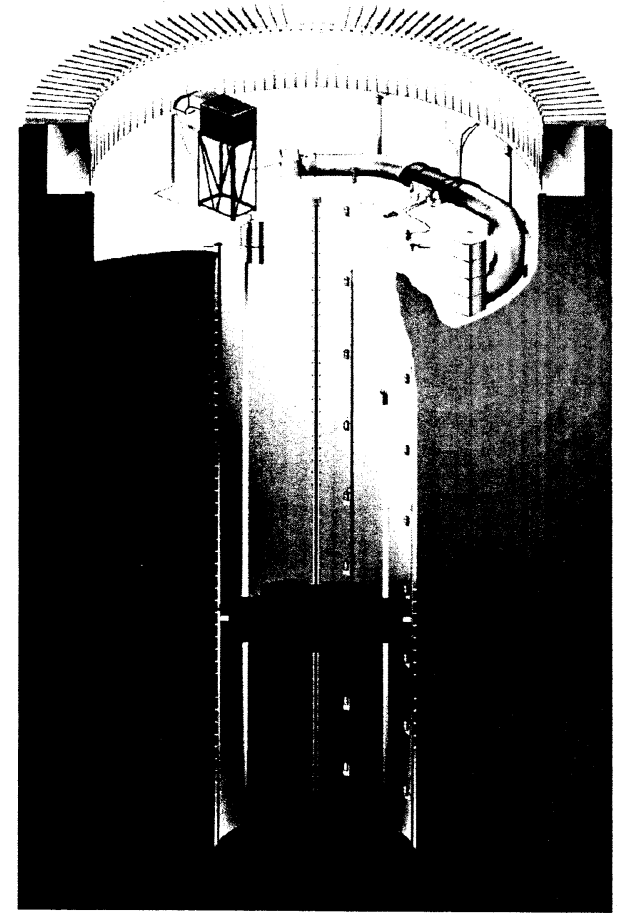

Fig.6 Deep-Sea Basin (NMRI).
The main features of the experimental riser model are presented in Table 1. Fig.7 shows the experimental riser model, its coordinate system is defined in the $x$-axis by the in-line motion, the $y$-axis corresponds to the transverse motion and the $z$-axis is defined in the direction of the riser's axis as shown in Fig.1. At the water level there is no horizontal velocity component $(U=0)$. Fig.8 shows the details of the bottom support of the riser model.

Table 1 Characteristics of the Riser Model.

\begin{tabular}{|l|c|}
\hline Material & Polyoxymethylene \\
\hline Model length $(\mathrm{m})$ & 35 \\
\hline Outer diameter $(\mathrm{m})$ & 0.0160 \\
\hline Inner diameter $(\mathrm{m})$ & 0.0108 \\
\hline Density $\left(\mathrm{kg} / \mathrm{m}^{3}\right)$ & 1410 \\
\hline Young's modulus $(\mathrm{MPa})$ & 2.937 \\
\hline
\end{tabular}

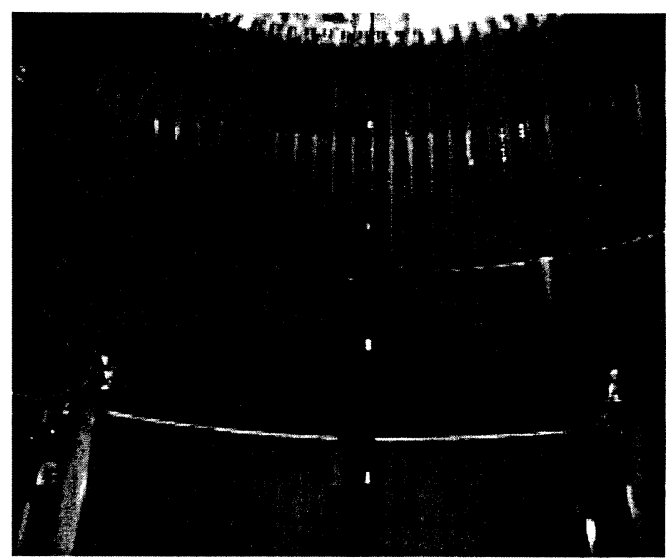

Fig. 7 35-meter Experimental Riser Model (NMRI).

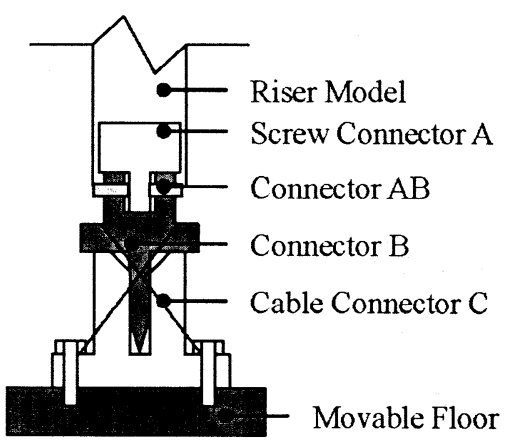

Fig.8 Bottom Connection of the Riser Model.

The riser model is fixed at its top end and along the $x$-axis is sinusoidal excited with an amplitude of $0.02 \mathrm{~m}$ (AX0) and periods ranging from 1.06 seconds to 1.56 seconds. Steel bars are added to the riser model in order to increase its self-weight. The total weight of the riser, including the steel bars, is $119.25 \mathrm{~N}$. 


\subsubsection{Numerical Simulation}

The FE model of the 35-meter flexible riser is assembled using 138 pipe elements; its self-weight is applied during the static step and hydrodynamic forces during the dynamic step as previously described. The model is fixed at its top end and the boundary condition at the bottom end, as shown in Fig.8, is numerically represented by a system of linear springs, which constrain displacements and moments in $x$-and $y$-axis, an additional linear spring constrains the $z$-axis moment. A nonlinear spring is additionally used to allow vertical displacements up to a maximum of $2 \mathrm{~mm}$ in the downward direction; in the upward direction this nonlinear spring restrains axial displacements.

Although the model is sinusoidal excited at its top end, its dynamic response is transient due to a time-varying load. It takes approximately 6 seconds for the wave to completely excite the bottom of the riser; then, the steady response is achieved and all sections of the model are sinusoidal excited having different oscillating frequencies, amplitudes and phase angles. The initial unsteady response of the model has a big incidence in the calculation of the phase angles according to Eq. (2). A numerical procedure is implemented in the proposed numerical scheme using the top end of the model as a reference. The phase angle is then calculated using the time difference between the time required for each section of the model to achieve its maximum amplitude and the required time at the top end to achieve the same condition. This procedure was implemented in the in-house FORTRAN subroutine.

The KC numbers achieved for the riser model range approximately between 2 and 8 . The hydrodynamic coefficients depend on the Reynolds number and $\mathrm{KC}$ number. The assumption that the hydrodynamic coefficients only depend on the $\mathrm{KC}$ number is completely avoided in this numerical implementation, at low $\mathrm{KC}$ numbers the beta parameter $(\beta=\mathrm{Re} / \mathrm{KC})$ must be considered. Lin et al. ${ }^{14)}$ present experimental data at $\beta=70$ and Obasaju et al. $\left.{ }^{6}\right)$ at $\beta=196$, which are the lower and upper limits, respectively of the range of the beta values achieved for the 35-meter riser model.

Obasaju et al.6) carried out a comprehensive study using circular cylinders in oscillating flow. In this experimental study it was demonstrated that in the transverse regime, $(8<\mathrm{KC}<15)$ the maximum lift coefficient is estimated to be 0.5 . Sanghafian et al. ${ }^{13)}$ numerically show a sharp decrease in the values of the maximum lift coefficients at low $\mathrm{KC}$ numbers $(2<\mathrm{KC}<8)$. Using a fixed beta parameter of 1035 , the computed maximum lift coefficient, $C_{\text {Lmax }}$, is 3.0 at $\mathrm{KC}=8$, but when $\mathrm{KC}=4$ and $\mathrm{KC}=2$, the maximum lift coefficients are $C_{L \max }=0.5$ and $C_{L \max }=0.1$, respectively. Considering that $70 \%$ of the pipe elements of the riser model are excited at very low $\mathrm{KC}$ numbers $(2<\mathrm{KC}<4)$ a conservative value of 0.5 for the maximum lift coefficient is used for the numerical validation of the dynamic scheme.

The natural frequencies of the numerical model were computed considering the added-mass effect. The reduced velocity of each section is checked and when its value is located in between 4 and 8 , lock-in is included in the simulation by switching the dominant frequency to the nearest natural frequency in Eq. (2) and increasing the mean drag coefficient according to the procedure previously presented. An average value of increased mean drag coefficient is used for each section, these values mainly ranges from 2.25 to 4 .

The damping force acting on a flexible riser is due to structural damping and fluid damping. In water structural damping will often be relatively small compared to fluid damping, and may therefore be of secondary influence. The damping force depends on the oscillation amplitude at frequencies above the lock-in region than at frequencies below the lock-in region. Within the lock-in region damping is frequency and amplitude dependent.

When a flexible riser is oscillating in the lock-in region, in addition to the damping force, the oscillating amplitude is also influenced by the magnitude of the mass ratio. The mass ratio is related to the ratio of the riser density to the fluid surrounding it. A decreasing value of the mass ratio induces increasing dynamic response as reported by Chakrabarti ${ }^{15)}$. Large-amplitude vibrations due to lock-in and a low mass ratio value are the main characteristics of the long flexible riser model presented in this paper; therefore, a structural damping ratio of $2 \%$ was included in the simulation scheme using as a reference the structural damping ratios proposed by Yamamoto et al. ${ }^{16)}$, which ranges between $2 \%$ and $5 \%$.

It is also important to highlight that during lock-in, the riser vibration is only limited by its structural damping. However, once the amplitude reaches about 1 or 1.5 times the riser's diameter, its vibration becomes self-limiting. If the riser does not achieve the lock-in condition, its vibration does not account significant amplification; this is the main reason why structural damping was not considered for the free-end riser models previously presented.

Fig.9, 10, and 11 show the time history response of the riser during 20 seconds exited at a period of $1.06 \mathrm{sec}$. and Fig.12, 13, and 14 at a period of $1.56 \mathrm{sec}$. In-line and transverse responses were computed at depths of $5 \mathrm{~m}, 10.5 \mathrm{~m}, 15 \mathrm{~m}, 20$ $\mathrm{m}, 25 \mathrm{~m}$ and $27.5 \mathrm{~m}$. 

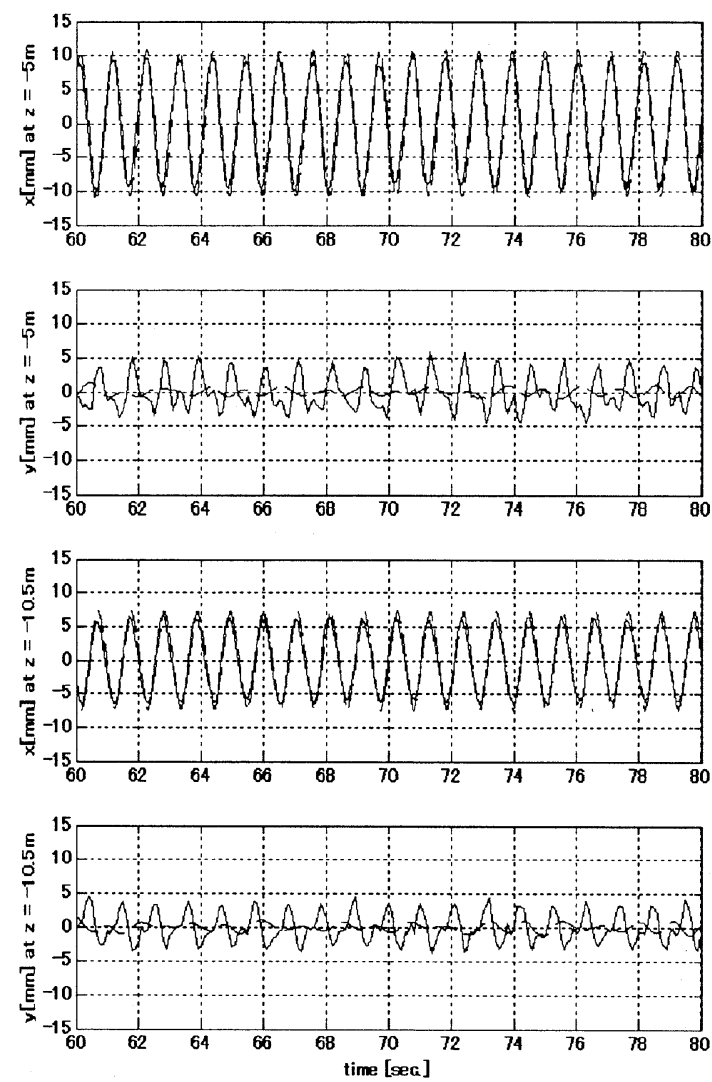

Fig.9 Time History Response at $z=-5 \mathrm{~m}$ and $z=-10.5 \mathrm{~m}$ $(T=1.06 \mathrm{sec}$. $)$.

--- Simulation - Experiment
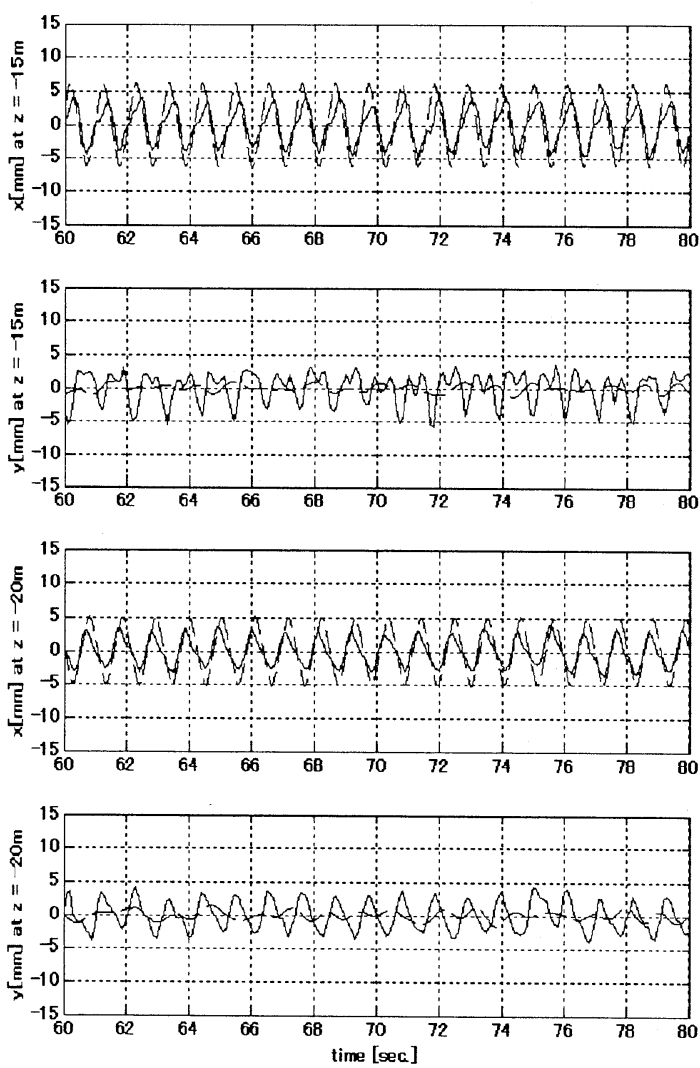

Fig.10 Time History Response at $z=-15 \mathrm{~m}$ and $z=-20 \mathrm{~m}$ $(T=1.06 \mathrm{sec}$. $)$.

--- Simulation - Experiment
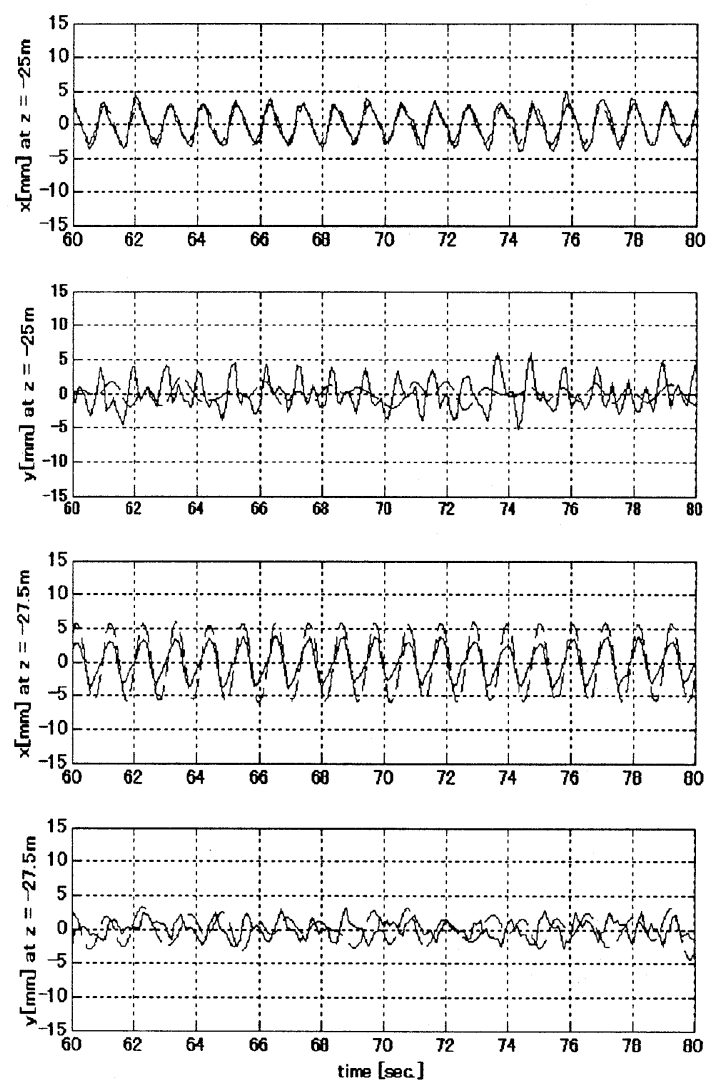

Fig.11 Time History Response at $z=-25 \mathrm{~m}$ and $z=-27.5 \mathrm{~m}$ $(T=1.06 \mathrm{sec}$. $)$.

--- Simulation - Experiment
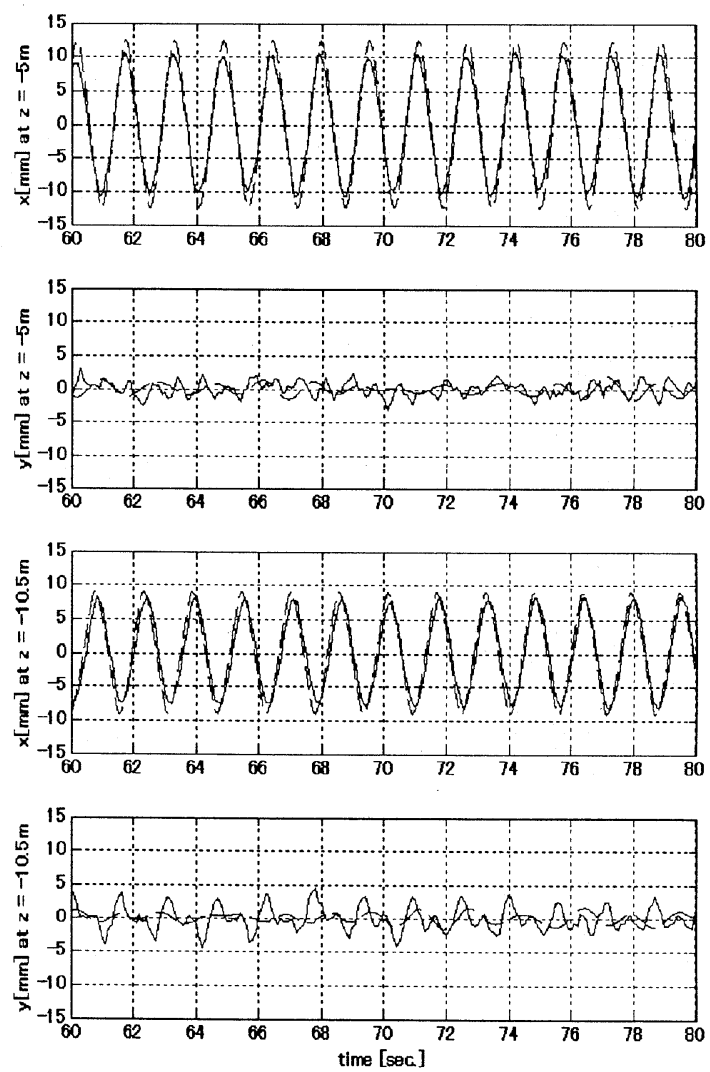

Fig.12 Time History Response at $z=-5 \mathrm{~m}$ and $z=-10.5 \mathrm{~m}$ $(T=1.56 \mathrm{sec}$. $)$

---- Simulation - Experiment 

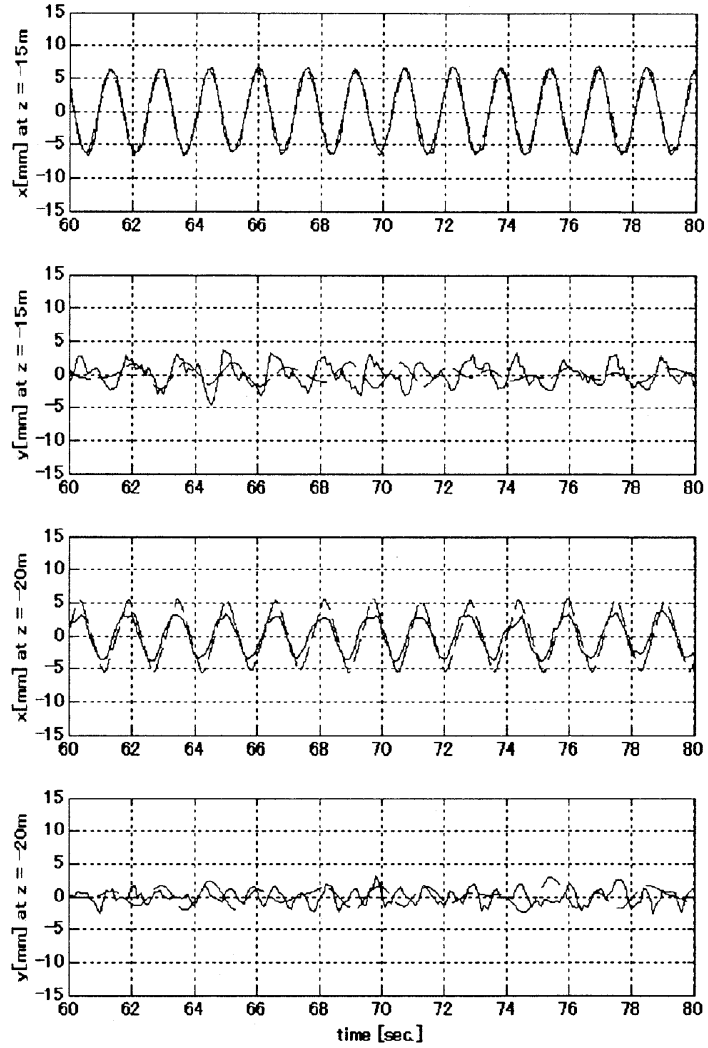

Fig.13 Time History Response at $z=-15 \mathrm{~m}$ and $z=-20 \mathrm{~m}$ $(T=1.56 \mathrm{sec}$.).

---- Simulation $\longrightarrow$ Experiment
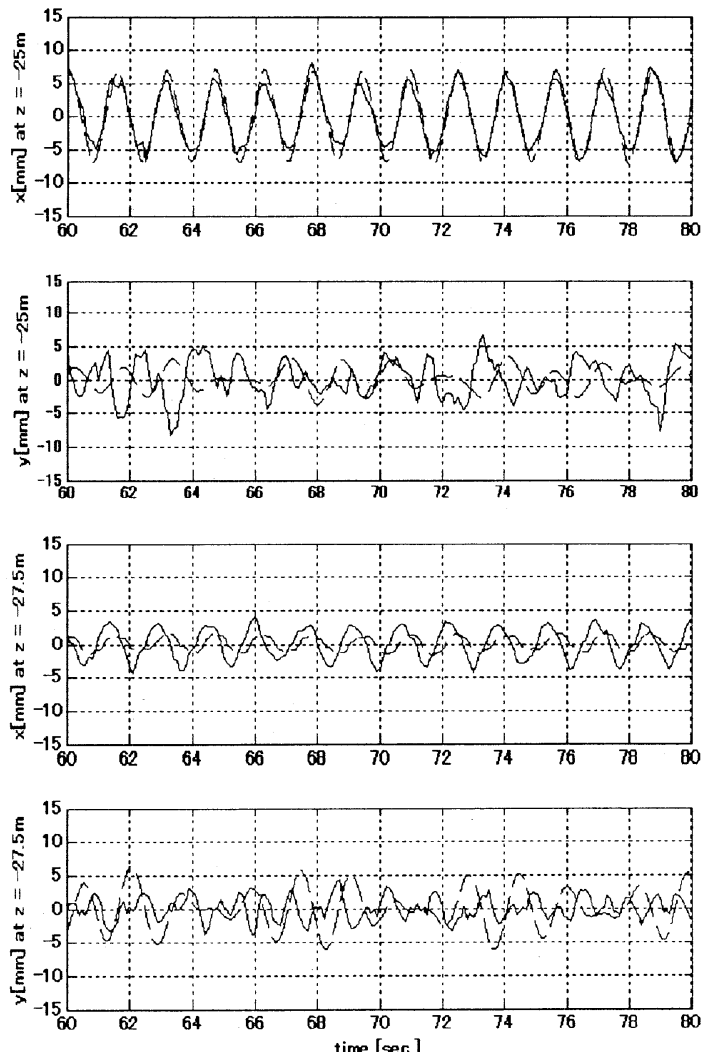

Fig.14 Time History Response at $z=-25 \mathrm{~m}$ and $z=-27.5 \mathrm{~m}$ $(T=1.56 \mathrm{sec}$. $)$.

--- Simulation - Experiment
The experimental data were passed through a $6^{\text {th }}$ order high-pass Butterworth filter with a $0.1 \mathrm{~Hz}$ cutoff. The in-line phase angles were corrected in order to improve the quality of the graphical results. Variations in the phase angles were found when the experimental results are compared with simulation results. These variations may be caused in part by the initial unsteady response of the riser.

Large displacements at the bottom end may be less dominant in the real boundary condition. Furthermore, although a proportional-type damping ratio of $2 \%$ was included in the simulation scheme, a local damping mechanism is likely to be acting at the bottom end and its numerical simulation may involve highly nonlinear behavior.

There is a considerable deviation in the transverse response in Fig.9, 10 and 11. The transverse response of the riser is under-predicted by the proposed numerical scheme when $T=1.06$ seconds; on the other hand, the in-line response of the riser presented in Fig.10 and 11, which correspond to the lock-out region, is over-predicted. The main reason for this tendency is that the transverse response of the riser has a big incidence in the mean drag coefficients, this fact has been proved by many experimental studies and even in the lock-out region the cross-flow amplitude may increase the mean drag coefficient and then reducing the in-line response.

In Fig.15 and 16 the maximum FFT amplitudes are computed for the in-line and transverse response of the riser. Good agreement is observed between the experimental and the simulation results. The transverse response of the model is relatively well predicted by the quasi-steady model and although the proposed numerical scheme considers the effect of the increased mean drag coefficient during lock-in, it is still challenging to predict the dynamic response of a flexible riser in this stage. The period of excitation ( $T=1.06$ seconds) induces a dynamic response having 36 elements of the FE model moving under lock-in considerations; on the other hand, when the period of excitation is increased to $T=1.56$ seconds, the number of FE elements under lock-in considerations is 62 .

Several studies have been conducted using the shedding frequency to compute the transverse response. In the proposed numerical scheme the dominant frequency is used instead of the instant shedding frequency in order to represent the dynamic transverse response. Obasaju et al. ${ }^{6}$ showed that the dominant frequency mainly depends on the $\mathrm{KC}$ number for a low beta parameter. 

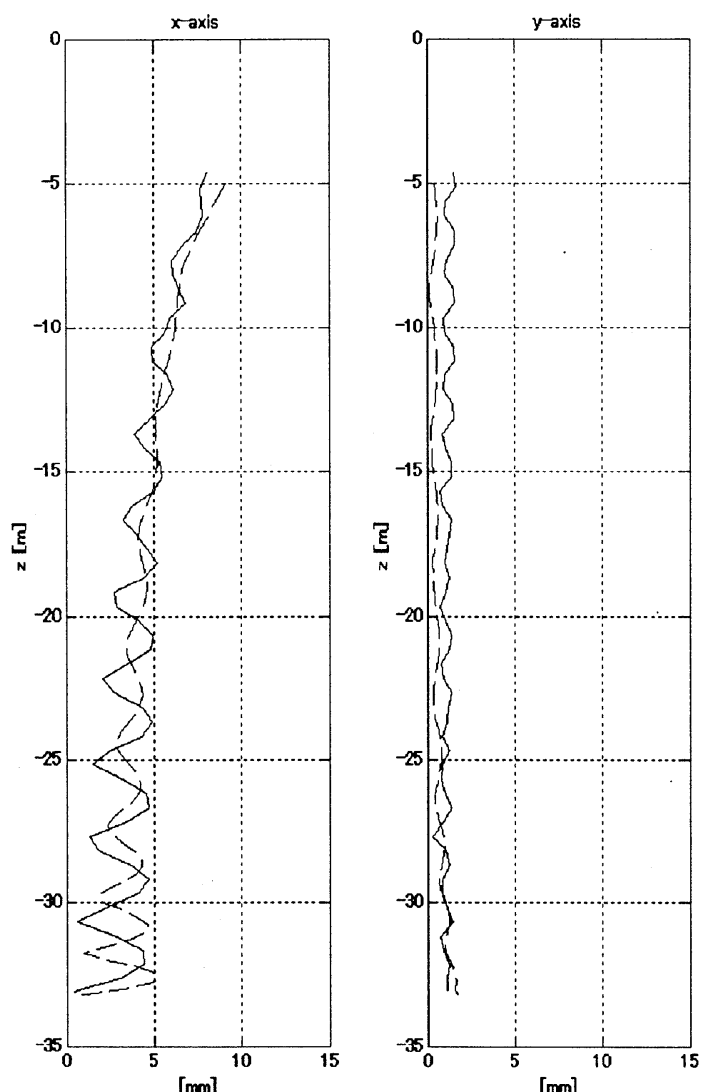

Fig.15 Maximum FFT Amplitudes ( $\mathrm{T}=1.06 \mathrm{sec}$.).

---- Simulation
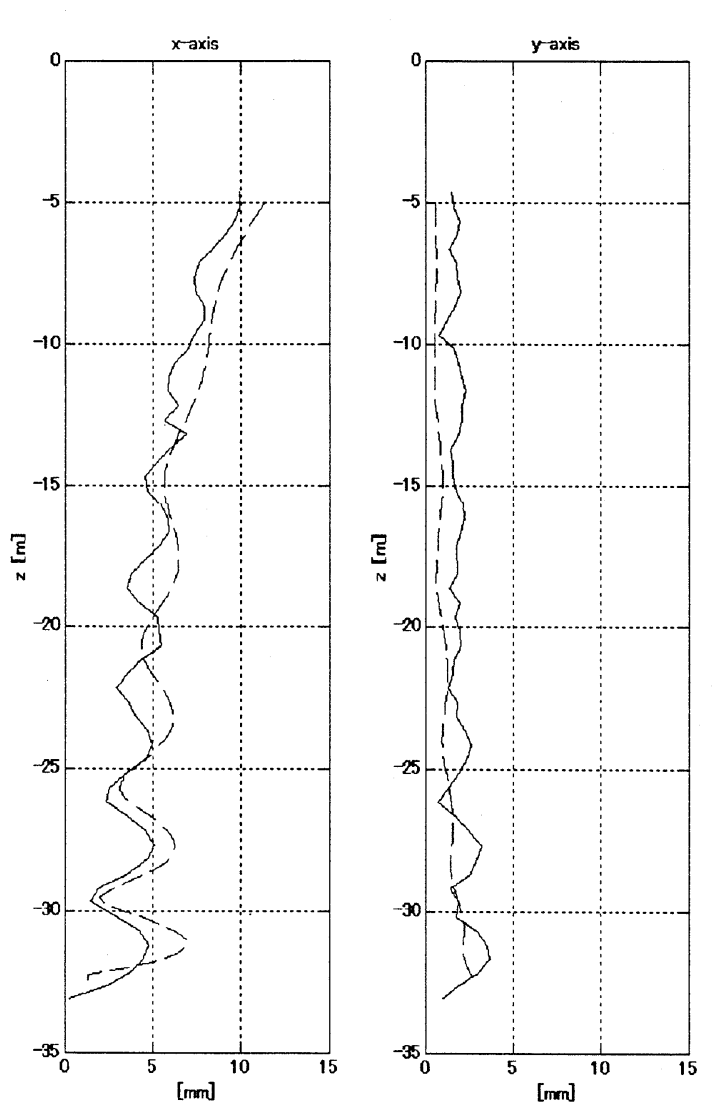

Fig.16 Maximum FFT Amplitudes ( $\mathrm{T}=1.56 \mathrm{sec}$.) -.-- Simulation
Experiment

Experiment
The in-line response is well represented by the proposed numerical scheme. There is a large region of the riser model located in the asymmetric region (mid section of the riser), where the lock-in condition is not achieved. In this region the mean drag coefficient is 1.5. According to Lin et al. ${ }^{14)}$ the asymmetric region shows a sudden drop in the mean drag coefficient when $\mathrm{KC} \approx 7$, that is not well represented by computational fluid dynamics. In addition, the values of the mean drag coefficient ranges from 1.7 to 1.4 for values of $\mathrm{KC}$ numbers located in between 2 and 7 .

The value of the mean drag coefficient in the mid section of the riser may have a big incidence in the dynamic response of the riser model. The value of 1.5 was kept in order to use a more realistic value. Furthermore, if the mean drag coefficient is increased proportionally to the cross-flow amplitude better results can also be achieved, but this approach must take into account large transverse amplitudes when the section of the riser is under lock-in conditions.

\section{Conclusions}

A numerical scheme to predict the dynamic response of deep-water risers was presented. Experimental data obtained from a 35-meter riser model was compared with the simulation results. One of the major advantages of the presented numerical scheme is that it can be used for long flexible risers due to its relatively simplicity when is compared with numerical schemes that involve the computation of the fluid forces using Computational Fluid Dynamics (CFD).

Good agreement is observed between simulation and experimental results. Two free-riser models were used to validate the in-line and the transverse response. The in-line response is accurately represented by the proposed numerical scheme and the transverse response also shows good agreement. At high oscillating frequencies there is a tendency of the proposed numerical scheme to under-predict the transverse response of the riser model.

It was found that the numerical model of boundary condition at the bottom end caused some errors in the dynamic response of the riser in regions located near the bottom end. The accurate modeling of a boundary condition is still one of the major challenges in the structural mechanics field. In this paper a system of linear and nonlinear springs was used to model the boundary at the bottom end. New modeling strategies must be considered in order to improve the quality of the dynamic response of the riser near the bottom end. 
The long flexible model due to its complex nonlinear behavior involves many challenges; the proposed numerical scheme using a numerical representation of the increased drag coefficient was also able to represent the main features of the dynamic response of the riser model.

An important issue to be considered in order to improve the numerical scheme presented in this paper; is the correct calculation of the hydrodynamic coefficients in the asymmetric region for this particular study. Although the values of these coefficients were taken from the best research work in that field, there are some modeling considerations that must be included in the numerical scheme, especially the segment of the 35-meter riser model located in the lock-out region. Further work using CFD must be carried out to improve the modeling considerations in this region.

Although VIV can occur in both steady currents and oscillating flow, only the oscillating flow case was presented in this paper. In steady currents, in the lock-in region, the VIV process may also induce large oscillating amplitudes in the riser as the reduced velocity is increased, but when the cross-flow amplitude reaches a certain value, the vortex shedding changes and then the cross-flow amplitude decreases. The oscillating flow case exhibits more complex behavior because the lock-in conditions can be achieved several times. As a result, the later case must be the core of the development of a dynamic response scheme for deep-water risers, which combines steady currents with oscillating flow.

This study shows a practical numerical scheme for the dynamic response of deep-water risers. The quasi-steady model can be easily incorporated in normal design practice under additional design considerations. The interaction between the steady current case and the oscillating flow case must be considered in further studies.

\section{REFERENCES}

1) Hong, Y. P. and Koterayama, W. : Numerical simulation scheme for dynamics of flexible riser and its validation by forced oscillation experiments, Int. Journal of Offshore and Polar Engineering, Vol. 14, No. 2, pp. 110-117, 2004.
2) Faccinetti, A. L., de Langre, E. and Biolley, F. : Coupling of structure and wake oscillators in vortex-induced vibrations, Journal of Fluids and Structures, Vol. 19, pp. 123-140, 2004.

3) Gabbai, R. D. and Benaroya, H. : An overview of modeling and experiments of vortex-induced vibration of circular cylinders, Journal of Sound and Vibration, Vol. 282, pp. 575-616, 2005.

4) Dütsh, H., Durst, F., Becker, S. and Lienhart, H. : LowReynolds number flow around an oscillating circular cylinder at low Keulegan-Carpenter numbers, Journal of Fluid Mechanics, Vol. 360, pp. 249-271, 1998.

5) Morison, J. R., O'Brien, M. P., Johnson, J. W. and Schaaf, S.A. : The force exerted by surface waves on piles, Petrol Trans. AIME, Vol. 189, pp. 149-154, 1950.

6) Obasaju, E. D., Bearman, P. W. and Graham, J. M. R. : A study of forces, circulation and vortex patterns around a circular cylinder in oscillating flow, Joumal of Fluid Mechanics, Vol. 196, pp. 467-494, 1988.

7) Pantazopoulos, M. S. : Vortex-induced vibration parameters: critical review, Offshore Technology ASME OMAE, Vol. 1, pp. 199-255, 1994.

8) Vandiver, J. K. : Drag coefficients of long flexible cylinders, Offshore Technology Conference OTC 4409, pp. 405-414, 1983.

9) Kim, W. J. and Perkins, N. C. : Coupled slow and fast dynamics of flow excited elastic cable systems, Joumal of Vibration and Acoustics, Vol. 125, pp. 155-161, 2003.

10) ABAQUS / Standard User's Manual, Version 6.5.

11) Senga, H. and Koterayama, W. : An experimental and numerical study on vortex-induced vibrations of a hanging flexible riser with its top in irregular motion, Int. Journal of Offshore and Polar Engineering, Vol. 15, No. 4, pp. 274-281, 2005.

12) Koterayama, W. and Nakamura, M. : Wave forces acting on a moving cylinder, Joumal of Offshore Mechanics and Arctic Engineering, Vol. 110, pp. 315-319, 1988.

13) Sanghafian, M., Stansby, P. K., Saidi, M. S. and Apsley, D. D. : Simulation of turbulent flows around a circular cylinder using nonlinear eddy-viscosity modeling: steady and oscillatory ambient flows, Journal of Fluids and Structures, Vol. 17, pp. 1213-1236, 2003.

14) Lin, X. W., Bearman, P. W. and Graham, J. M. R. : A numerical study of oscillatory flow about a circular cylinder for low values of beta parameter, Journal of Fluids and Structures, Vol. 10, pp. 501-526, 1996.

15) Chakrabarti, S. K. : The theory and practice of hydrodynamics and vibration, World Scientific Publishing, Singapore, 2002.

16) Yamamoto, C. T., Meneghini, J. R., Saltara, F., Fregonesi, J.A. and Ferrari Jr., J. A. : Numerical simulations of vortex-induced vibration on flexible cylinders, Journal of Fluids and Structures, Vol. 19, pp. 467-489, 2004.

(Received April 13, 2006) 